\title{
Transmission
}

\section{and Reception of the Translated Versions of San Yan Er Pai in the English World}

\author{
Shuang Qiu ${ }^{1, a}$, Ping $\mathrm{Yi}^{2, b^{*}}$ \\ ${ }^{1}$ Collegel of Foreign Languages, Chengdu University of TCM, Chengdu, Sichuan, China \\ ${ }^{2}$ College of Foreign Languages, Chengdu University of TCM, Chengdu, Sichuan, China \\ a78027668@qq.com \\ b*904026750@qq.com
}

\begin{abstract}
San Yan Er Pai is the peak of ancient vernacular novels in China. It is an important embodiment of the translation of Ming and Qing novels and cross-cultural communication between China and the West. This paper sorts out the transmission process of translations in the English world and analyzes the different reception situations and reasons of each one.

Keywords: San Yan Er Pai, translated versions, transmission, reader's recption
\end{abstract}

\section{“三言二拍” 英译本在英语世界的传播与接受分析 \\ 邱爽 $1, a$ 易平 $2, b^{*}$}

${ }^{1}$ 成都中医药大学外语学院, 成都, 四川, 中国

成都中医药大学外语学院, 成都, 四川, 中国

a78027668@qq.com

b*904026750@qq.com

\section{摘要}

“三言二拍” 作为中国古代白话小说的巅峰之作，是明清小说翻译和中西方跨文化交流的重要体现。 文章梳理英译本在英语世界的传播历程，对四个重要译本进行全面解读，并分析各译本不同的接受情 况和原因，为进一步促进中西方文化的广泛交流，为中国文化“走出去”做出贡献。

关键词：三言二拍，英译本，传播，读者接受

\section{1. 前言}

明代由冯梦龙编著的《喻世明言》、《警世通言》、 《醒世恒言》以及凌濛初撰作的《初刻拍案惊奇》、《二 刻拍案惊奇》总称 “三言二拍” , 被认为是我国古代白 话短篇小说的巅峰之作。自十六世纪第一次进入英语世 界, 经历了几个世纪的缓慢渐入, 终于从零星的选篇转 述进入到丰富的多元化译本, 还呈现出一些带有研究性 质的作品。随着明清小说在英语世界传播的跨越式发 展, “三言二拍” 也在不断被翻译和误读的复杂过程中, 逐步被西方学界和读者接受与肯定。

\section{2. “三言二拍” 的英译本介绍}

据今所知, 最早的 “三言二拍” 英译文是约翰 - 瓦 茨 (John Watts) 1736年转译法文版《中华帝国全志》里 的: 《庄子休鼓盆成大道》、《怀私怨狠仆告主》和《吕 大郎还金完骨肉》，它们也是中国小说中最早被译为西 文的作品。初期的译介是零散的、不成系统的, 更大的 目的不是作为中国古典文学的研究, 而是为了让外国人 学习中文, 或是更多了解中国文化。有些翻译者, 自己 并不能认识或者使用中文, 他们翻译的依据是其他语言 的翻译作品, 这样所引起的对原作的误读和曲解是不可 避免的。在这些作品中, 最为重要的是两位汉学家多马 斯当东(Sir George Thomas Staunton)和道格拉斯(R. K. 
世通言》的全译本Stories to Caution the world : a Ming

Douglas)的译本。多马斯当东曾经在1792年来到中国, 并且是当时唯一能用官话和乾隆皇帝谈话的英国人。他 还曾翻译过《大清律例》, 《异域录》, 并且有《多马 斯丹东回忆录》等著作。而道格拉斯的《中国故事》 里还收录了中国明清时另一位著名的小说家李渔的《夺 锦楼》The Twins, From the Chinese of Wu Ming。在此 后的半个世纪里, 国外汉学界对于中国文学的研究主要 集中在古典诗歌方面, 而对于古典小说这一部分甚为忽 略。

直到西里尔・伯奇(Cyril Birch)1958年编译出版 Stories from a Ming Collection, 让 “三言二拍” 的译本 开始具备学术取向。这本书中, 伯奇在每篇译文之前都 对故事的内容作了简短的介绍和评论, 而译文本身也更 完整, 包括了入话和诗词的部分, 并带有注释, 使得译 文更流畅、生动, 充分传达了原著的风貌, 非常具有可 读性。其中《吴保安弃家赎友》The Story of Wu Pao-an 一篇, 伯奇还同时译出了白话和文言两种版本[1]。这部 当时最有价值也是评价最高的译作流传至今, 甚至对于 后来的一些翻译作品, 起到了深远的影响。

2006年著名汉学家韩南（Patrick Hanan）教授出版 的经典译本Falling in Love: stories from Ming China在学 术和研究的价值方面都达到了顶峰。作者选取了两本明 朝小说《醒世恒言》和《石点头》中的七篇故事进行翻 译, 强调保留原作的完整性和语言韵味, 对涉及的典故、 诗词等都做有详细注释, 非常具有学术价值。韩南在序 言中, 对于书中的七篇故事分别作了介绍, 提及《莽书 生强图驾侣》The Reckless Scholar和《潘文子契合驾葟 冢》The Lovers' Tombs 两篇时, 特别对于它们的作者李 渔给与了很高的评价[2]。我们在强调韩南教授对于中国 明清小说的成就上, 他对于李渔作品的翻译和研究也是 很重要的一方面。他翻译的《十二楼》A Tower for the Summer Heat [3], 《无声戏》Silent Operas [4]以及 《肉蒲 团》The Carnal Prayer Mat [5]都具有极高的学术价值, 在英语世界广泛流传。韩南教授的翻译作品成就很高, 在宇文所安 (Peter France) 所编的Oxford Guide to Literature in English Translations 一书中, “把Patrick Hanan与David Hawkes, Anthony Yu, David Roy 并列, 认 为这四位学者, 翻译者大幅度提升了中国文学整体英译 的水平提供了忠实的全译版本, 保存了原作的风格与风 味。[6]”

华盛顿大学比较文学专业的中国留学生杨树辉和 杨云琴2000年编译Stories Old and New: A Ming Dynasty Collection Compiled by Feng Menglong(1574-1646)出版, 第一次完整收录了《喻世明言》的全部四十篇作品。这 部全译本延续了Story from a Ming Collection 的风格, 包 括有序言, 入话和诗词的部分。在序言里, 译者首先确 定了 “三言” 在明朝, 甚至中国通俗小说史上的里程碑 地位。然后分别介绍了 “三言” 的成书的年代、冯梦龙 的生平以及其他作品。然后, 还指出了 “三言” 在编排 上的特点一一成双成对的故事(pared stories), 以及就“三 言” 中讲述者的修辞(the storyteller's rhetoric)等进行了分 析[7]。从序言中, 我们看出了作者对于 “三言” 的情有 独钟, 可能也正是这个原因, 后来又在 2005 年出版了《警
Dynasty Collection Volume 2 [8]和《醒世恒言》的全译本 Stories to Awaken the World: A Ming Dynasty Collection Volume 3[9]。这套书也代表着英语世界的 “三言二拍” 进入到全面丰收的时期, 对整个中国古典白话小说的译 介和研究起到了巨大的推动作用。

除了汉学家和学者们的努力, 来自民间的翻译力量 也为 “三言二拍” 的传播做着贡献。旅居美国的华人王 惠民(Ted Wang)和陈陈(Chen Chen)2007年选取了 “三 言” 中的八个故事进行翻译, 出版发行The Oil Vendor and the Courtesan Tales from the Ming Dynasty。在序言 中, 作者提到之前一部的翻译作品The Abbot and the Widow: Tales From the Ming Dynasty 是从 “二拍” 中选取 了十个故事 $[10]$ 。两位作者都不是中文或英语专业, 也 没有关于汉学的研究经历, 他们的翻译目的是希望能够 让读者认识到中国文学轻松有趣的另一面, 并期望唤起 对于中国文学的兴趣。这部译作并不避讳使用直译的方 式来重现中文里特有的词语，也没有运用大量的英文习 语来置换原文的修辞，这些 “不可译” 的成分，恰好成 为了译者增进故事趣味的方法, 让这部译文更有中国氛 围。关于原文中第一人称的称呼, 为了配合中文的说话 习惯，王惠民和陈陈没有全部翻译为 “I” 而使用了 “this humble person” [10], “this small person” [10]这样的 自称, 译出了原文中谦卑的语气。而对于中文里的长度, 货币, 时间等单位的表达方式, 也全数保留。译者以拼 音 “mu” 来表示 “亩”，用 “tael” 来计算价格，也以 阴历 “lunar month” 来表达时间。正是这种不同于学者 的自由的翻译方式, 使这部译本语言独特, 富于趣味, 在英语世界里的 “三言二拍” 翻译史上独树一帜。

\section{3. 国内外 “三言二拍” 英译本研究}

对以“三言二拍”为例的中国明清小说的英译研究， 西方研究者已经从语言学视角的翻译研究转向以跨学 科的视角认识翻译现象。他们将翻译视为发生在一定背 景下的跨文化交流活动, 认识到翻译受译者、读者及其 他多方面影响。韩南 (Patrick Hanan) 教授的一系列重 要研究论文: The Authorship of Some Ku-Chin hsiao-shuo Stories (1969) [11], The Making of The Pearl-Sewn Shirt and The Courtesan's Jewel Box (1973)[12], The Chinese Short Story: Studies in Dating, Authorship, Composition (1973) [13], The Chinese Vernacular Story (1989) [14] 对 “三言二拍” 的写作年代、地点、作者以及写作风格、 特色等都进行了深入研究。美国比较文学教授何谷理

（Robert E. Hegel）也运用不止一个专业的视角研究“三 言二拍” 以及其他明清小说在接受者辨析、明清小说插 图、点评以及小说外形特征与读者接受的关系等方面取 得重要成果[15]。

国内对于英语世界的“三言二拍”研究起步较晚, 大 多从翻译、传播和译者等方面展开。著名翻译家杨宪益 和戴乃迭的Stories from the Ming Dynasty（1957）[16]成 为国内最重要、相关研究最丰富的译本。有关于译本的 


\begin{tabular}{|c|c|c|c|c|}
\hline & $\begin{array}{c}\text { Dynasty } \\
\text { Collection } \\
\text { Volume } 2 \\
\end{array}$ & Press, 2005 & & \\
\hline 5 & $\begin{array}{c}\text { Stories to } \\
\text { Awaken the } \\
\text { World: A } \\
\text { Ming } \\
\text { Dynasty } \\
\text { Collection } \\
\text { Volume } 3 \\
\end{array}$ & $\begin{array}{c}\text { Seattle: } \\
\text { University } \\
\text { of } \\
\text { Washington } \\
\text { Press, 2009 }\end{array}$ & 302 & 10 \\
\hline 6 & $\begin{array}{l}\text { The Oil } \\
\text { Vendor and } \\
\text { the } \\
\text { Courtesan } \\
\text { Tales from } \\
\text { the Ming } \\
\text { Dynasty }\end{array}$ & $\begin{array}{c}\text { New York, } \\
\text { NY: } \\
\text { Welcome } \\
\text { Rain, } 2007 .\end{array}$ & 76 & 7 \\
\hline
\end{tabular}

文化负载词、俗浐语和诗词的翻译研究, 以及从改写理 论视角、关联理论视角和翻译伦理学视角研究译本。关 于国外译者的研究, 集中在西里尔・伯奇和韩南的翻译 策略方面。在传播领域, 王丽娜 (1984) 最早展开 “三 言二拍” 的海外传播研究, 以文献罗列的形式梳理海外 藏本、翻译及研究著作[17]。王平（2006）用传播学理 论对包括 “三言二拍” 的明清小说从传播者、传播媒介、 传播内容、受众和传播效果等部分进行研究 [18]。邱爽 （2017）总结了其在英语世界的传播, 并增补和研究了 二十世纪以后的译作 [19]。近年来对中国文学的翻译研 究呈现出新的方法和视角, 例如运用语料库的方法研究 译本词语、译者风格和翻译策略等; 从翻译生态学视域、 跨文化视角以及副文本角度等解读译本。在对外传播方 面, 不再只关注英译本的数量, 而开始调查译本在海外 的接受情况, 帮助中国文化 “走出去” , 成为世界文化 中的独特组成部分。

\section{4. “三言二拍” 英译本的接受情况和影响}

研究目的语读者的可接受性是一个非常重要的、持 续不断的动态过程, 也是未来翻译研究的趋势。目的语读 者对译本的可接受性, 可以帮助译者使用正确的翻译策 略, 缩小和读者的差异。而且研究目的语读者的可接受 性是个不断变化的过程, 因为不同时代的人对同一译本 的可接受性是不一样的, 他们对待翻译作品的态度是不 一样的。

英语世界见证了 “三言二拍” 走向世界的一次次不 懈尝试, 笔者选取上文提到的重要译本, 从实际情况和 可操作性出发, 借助Worldcat数据库资料调查译本在英 语世界各图书馆的馆藏情况, 以及公开发表的评论文章 和目的语读者评价, 试图更全面、真实地了解 “三言二 拍”英译本在西方的接受情况。

通过搜索得到藏有“三言二拍” 各个英译本的英语 世界图书馆分布情况（参见表1）。

\section{表 1 藏有 “三言二拍” 不同英译本的英语国家图书 馆数量统计表}

\begin{tabular}{|c|c|c|c|c|}
\hline 序号 & 书名 & 版本 & $\begin{array}{c}\text { 收藏该译 } \\
\text { 本的美国 } \\
\text { 图书馆数 } \\
\text { 量 }\end{array}$ & $\begin{array}{c}\text { 收藏该译本 } \\
\text { 的其他英语 } \\
\text { 国家图书馆 } \\
\text { 数量 }\end{array}$ \\
\hline 1 & $\begin{array}{l}\text { Stories from } \\
\text { a Ming } \\
\text { Collection }\end{array}$ & $\begin{array}{c}\text { New York: } \\
\text { Grove Pr. } \\
1958\end{array}$ & 9 & 9 \\
\hline 2 & $\begin{array}{l}\text { Falling in } \\
\text { Love: } \text { stories } \\
\text { from Ming } \\
\text { China }\end{array}$ & $\begin{array}{c}\text { Honolulu: } \\
\text { University } \\
\text { of Hawai'i } \\
\text { Press, } 2006 .\end{array}$ & 380 & 40 \\
\hline 3 & $\begin{array}{c}\text { Stories Old } \\
\text { and New: A } \\
\text { Ming } \\
\text { Dynasty } \\
\text { Collection }\end{array}$ & $\begin{array}{c}\text { Seattle: } \\
\text { University } \\
\text { of } \\
\text { Washington } \\
\text { Press, } 2000\end{array}$ & 278 & 21 \\
\hline 4 & $\begin{array}{l}\text { Stories to } \\
\text { Caution the } \\
\text { world : a } \\
\text { Ming }\end{array}$ & $\begin{array}{c}\text { Seattle: } \\
\text { University } \\
\text { of } \\
\text { Washington }\end{array}$ & 235 & 18 \\
\hline
\end{tabular}

数据显示 “三言二拍”英译本的收藏主要分布在美 国各高校的图书馆，而其他英语国家则较少。Stories from a Ming Collection虽然年代久远数量最少, 依然有 超过 10 个国家的图书馆收藏, 从一个方面也体现出译本 的价值, 甚至对于后来的一些翻译作品起到了深远的影 响。

Falling in Love: stories from Ming China 是所有“三 言二拍” 英译本中馆藏数量最多、范围最广的作品。他 深厚的学术背景, 以及对中国文化特别是中国白话小说 研究的突出成绩, 都吸引着中国文学专业的美国大学 生, 并将其视为美国学术教科书。韩南作为学术界的代 表, 首先根据自己的专业发展需要开始接触中国古典文 学, 带着交流、互补和发展的目的去主动学习异质文化。 然后通过辨别、选择, 过滤出自己感兴趣的中国古典白 话小说做进一步研究, 最后把自己的研究与中国白话小 说有机的结合起来。译本的目标读者是研究中国文学的 学者和学习中国文化、文学的学生, 翻译目的是增加在 国外的中国文学教材的选择性。译本读者对于“三言二 拍”的接受, 也是一种接受者因文化需要而主动向发送 者文化学习, 并选择适合于自身文化发展的内容。

杨树辉和杨云琴的 “三言” 全译本收录也很广泛, 被学者们认为是“一个重要的对亚洲文学的翻译和中国 历史的补充”。Review of Bibliography in Sinology评价为 "As a truly complete collection of vernacular stories, [this volume] clearly sets a new standard for the English-speaking world."译本还入选了《大中华文库》, 并由岳麓书社出版发行了英汉对照本, 为推进“中国文 化走出去” 起到了积极作用。

译本The Oil Vendor and the Courtesan Tales from the Ming Dynasty在亚马逊等美国主流购书网站的浏览 和购买量说明其目标读者更加广泛，包括亚裔后代、对 亚洲或中国感兴趣的大众以及一般对于阅读有兴趣的 普通读者。

\section{5. 结语}

“三言二拍” 作为中国古代白话小说的巅峰之作, 在对外传播的过程中展现出中国文化独特的鬼力和价 值。本文通过分析英译本在英语世界的传播历程和接受 
[12] Patrick, H.(1973) The Making of The Pearl-Sewn Shirt and The Courtesan's Jewel Box, Harvard Journal of Asiatic Studies, Harvard University Press, Volume 33:124-153.

[13] Patrick, H.(1973) The Chinese Short Story: Studies in Dating, Authorship, Composition, Harvard University Press, Cambridge

[14] Patrick, H.(1981) The Chinese Vernacular Story, Harvard University Press, Cambridge.

[15] Robert E. H (2014) The Colloquial Short Story in China: A Study of the San-yen Collections, Harvard University Press, Cambridge.

[16]Yang, XY(1957) Stories from the Ming Dynasty, Beijing Foreign Languages Press, Beijing.

[17] Wang, LN(1984) Overseas Collection, Foreign Language Translation and Research Works, Journal of Chinese Literature and History,Volume1:180-183

[18] Wang, P(2006) A Study on the Dissemination of Vernacular Novels in China, Shandong Education Press,Jinan.

[19] Qiu,S. (2017) A Comparative Study of Translator's Style in the Two English Versions of San Yan Er Pai, Journal of Chengdu Normal University, Volumel: 2017.6:61-64
[5] Patrick, H.(1990) The Carnal Prayer Mat, Ballantine Book, New York.

[6]France, P. (2001) Oxford Guide to Literature in English Translations. Oxford University Press, Oxford.

[7] Yang,SH, Yang YQ.(2000), Stories Old and New: A Ming Dynasty Collection Compiled by Feng Menglong(1574-1646), The University of Washington Press, Washington.

[8] Yang,SH, Yang YQ.(2005) Stories to Caution the world : a Ming Dynasty Collection Volume 2 compiled by Feng Menglong, The University of Washington Press, Washington.

[9] Yang,SH, Yang YQ.(2009) Stories to Awaken the world : a Ming Dynasty Collection Volume 3 compiled by Feng Menglong, The University of Washington Press, Washington.

[10] Wang,T ,Chen,C.(2007) The Oil Vendor and the Courtesan Tales from the Ming Dynasty, Welcome Rain Publishers, New York.

[11] Patrick, H.(1969) The Authorship of Some Ku-Chin hsiao-shuo Stories, Harvard Journal of Asiatic Studies, Volume 29:30-38 